\title{
Érasme, les intellectuels et l'affaire Reuchlin
}

DANIEL MÉNAGER

Summary: Intellectuals have been more than once accused of not caring about world affairs. Now, Erasmus appears to announce a modern reflection on power and knowledge. An archetype of our contemporary intellectual, he looked at and criticized the society he lived in. As a theologian and a priest, he conferred sacerdotal authority on his speech, but he spoke for a large audience, not exclusively for scholars. As a famous man, he played a role in the "Reuchlin affair," the first time that intellectuals made a public engagement. Without blindly following the "Reuchlinist party," Erasmus defended a scholar with his name and his pen. But he trusted the civil authorities more than pure truth, and he refused to discredit the theologians.

e nos jours, en Europe occidentale, l'intellectuel n'a pas bonne presse. On lui reproche, pêle-mêle, d'être conservateur et critique, de trancher de tout sans posséder les compétences techniques lui permettant de parler à bon escient, et de se mêler de ce qui ne le regarde pas. Et pour faire bonne mesure, on prédit à plus ou moins longue échéance sa disparition pure et simple. Le vent de populisme qui souffle actuellement sur cette partie du monde n'arrange pas ses affaires. L'intellectuel ? Il ne va pas sur le «terrain », un mot devenu sacro-saint dans la bouche de certains politiques, il ne connait pas les « réalités » d' "en-bas ». Bien entendu, les auteurs de ces critiques font semblant d'oublier que certains intellectuels sont allés courageusement en Afghanistan ou au Kosovo pour voir de leurs yeux ce qui s'y passait. À chacun sa réalité. Celle de nos modernes populistes est inséparable des vieux villages de la France, ou, dans le meilleur des cas, des quartiers difficiles de nos grandes villes.

Ces reproches ne datent pas d'aujourd'hui. Sartre les examinait déjà dans les trois conférences qu'il fit au Japon en 1965 et qui furent publiées sous le titre : Plaidoyer pour les Intellectuels ${ }^{1}$. On pouvait les lire déjà sous la plume des adversaires de Dreyfus, qui inventèrent le mot. L'intellectuel 
avait le grand tort, en particulier, de s'occuper de ce qui ne le regardait pas ${ }^{2}$. S'il appartenait à la science, qu'il reste un savant. À l'Église, qu'il demeure un clerc. À l'Université, qu'il s'occupe de la date de naissance des écrivains et des éditions de ses œuvres. Il est même probable que, sous des formes différentes, bon nombre des reproches qui viennent d'être résumés sont formulés bien avant. Ce qui pose la question de l'époque où apparaissent les intellectuels. Le XVIII ${ }^{\mathrm{e}}$ siècle ? On l'a souvent écrit, non sans raison d'ailleurs. Peut-on remonter plus haut sans tomber dans le «péché d'anachronisme $»^{3}$ ? Qu'il ait été commis par un de nos plus grands historiens, Jacques Le Goff, qui est aussi un véritable intellectuel, donne une idée des audaces nécessaires pour mieux comprendre l'histoire. Pour lui, en effet, il y eut des intellectuels au Moyen Âge ${ }^{4}$, c'est-à-dire des clercs « qui font métier de penser et d'enseigner leur pensée $»^{5}$. Ils ne dédaignent pas la bataille des idées et ne croient pas indigne d'eux de descendre dans l'arène pour ou contre Aristote, pour ou contre Abélard, pour ou contre le Roman de la Rose. Le lieu de leur activité, c'est la ville, qui connaît à partir du XII ${ }^{\mathrm{e}}$ siècle un essor sans précédent. Époque bénie, si l'on en croit l'éminent médiéviste, car tout ou presque va changer avec la Renaissance ${ }^{6}$. Fini le tumulte des Universités et les furieuses empoignades des clercs. L'humaniste de la Renaissance n'aspire qu'à la quiétude. Regardons-le avec A. Chastel ${ }^{7}$ dans son studiolo, entouré de ses livres, gardé par un chien somnolent ou, quand il s'incarne en saint Jérôme, par un lion inoffensif, ne levant les yeux de la page qu'il lit, de la feuille où il écrit que pour suivre dans son esprit la trajectoire de ses pensées, ou, à l'extérieur, le vol d'un oiseau. Baudelairien avant la lettre, il aime le luxe et le calme, parfois peut-être, la volupté. Qu'irait-il faire dans la fureur du monde?

Érasme, lui aussi, aimait la quiétude, et l'otium des lettrés. Il fut pourtant le plus grand intellectuel de son époque, c'est-à-dire quelqu'un qui refusa de se laisser enfermer dans une spécialité et crut de son devoir de se mêler de ce qui, à première vue, ne le regardait pas. Pourtant, cet engagement présente de singulières ambiguïtés.

\section{I}

Érasme était devenu, à une date assez tardive, docteur en théologie ${ }^{8}$. J. Chomarat précise : « dans une université de second ordre ${ }^{9}$. Selon lui, il n'a jamais accordé grande importance à ce titre. De fait, on ne manque pas de textes qui justifient la thèse du grand spécialiste d'Érasme, celui-ci par exemple qui ne lui a pas échappé : "Il n'y a rien dont je fasse moins profession que d'être théologien, même si jadis, sous la pression de certains amis, je me suis enrôlé dans les rangs des théologiens. Où m'a-t-on vu dans mes ouvrages me targuer du titre de théologien. Qui m'a jamais entendu me 
flatter de ce titre, même dans une conversation familière ? ${ }^{10}$. Dans le meilleur des cas, le théologien construit des systèmes. Souvent, il s'occupe de vétilles qui ne font pas progresser l'amour de Dieu. Érasme se veut avant tout philologue, il scrutera jusque dans le détail la lettre de l'Écriture, mais, quand il s'agit du Verbe divin, il n'y a pas de détails. Il veut donc, pour reprendre encore les idées et les mots de J. Chomarat, favoriser une renaissance de la théologie vraie, "fondée sur la connaissance de l'Écriture et servante de la piété ${ }^{11}$.

Il lui arrive pourtant de revendiquer non le titre, mais le discours du théologien. L'un des exemples les plus intéressants se trouve dans la lettrepréface de l'Institution d'un prince chrétien (mars 1516), où on lit ceci : «Moi [...] qui suis un théologien, je veux un prince glorieux et d'une parfaite intégrité ». La phrase s'explique d'abord par référence aux auteurs de l'antiquité qui viennent d'être cités et qui se sont efforcés de tracer le portrait du prince idéal. On peut faire mieux, semble dire Érasme, quand on adopte le point de vue de la théologie chrétienne. La suite de la phrase est pourtant déconcertante : «En outre, je suis un chrétien, et veux un prince chrétien ${ }^{12}$. Que veut dire ce « en outre »? À prendre les choses trop à la lettre, on serait tenté de penser que tous les théologiens ne sont pas vraiment chrétiens, ce que laissaient déjà entendre certaines pages peu amènes de l'Éloge de la Folie. Mais ici, c'est Érasme qui parle sans masque. On pourra lever une partie de la difficulté en remarquant que dans ce passage, comme ailleurs ${ }^{13}$, Érasme revendique un point de vue plus large que celui du théologien. C'est un peu dans ce sens qu'il ose écrire, dans l'Exhortation: «Celui qui sous l'inspiration du Christ prêche ces vertus [celles de l'Évangile] et d'autres semblables, qui les enseigne, y exhorte, y invite, y anime, celui-là seul est authentiquement théologien, même s'il est terrassier ou tisserand ${ }^{14}$. De quoi faire hurler tous ceux qui étaient fiers de leur savoir technique ! On pourrait penser, également, que ce point de vue n'est pas très éloigné de celui de Luther. Il s'en faut pourtant de beaucoup. On ne peut oublier en effet les termes très connus dans lesquels Érasme, écrivant à Paul Volz ${ }^{15}$, présente les hiérarchies des sociétés chrétiennes. Au centre du monde, le Christ, entouré de nombreux cercles. On trouve dans le premier, le plus proche de la personne divine, les prêtres, les évêques, les cardinaux, les pontifes, « tous ceux auxquels il appartient de suivre l'Agneau partout où il va ». Réunis dans un second cercle, les princes profanes servent le Christ, à leur façon, avec les armes et les lois. «Dans le troisième cercle nous placerons le commun peuple, en tant que partie la plus grossière de ce globe, mais non point si grossière qu'elle n'appartienne au corps du Christ ${ }^{16}$. Le schéma serait statique si chaque catégorie n'attirait près du Christ celle qui est placée au-dessous d'elle. Un peu comme cela se passe 
dans les Hiérarchies divines du pseudo-Denys ${ }^{17}$. Il n'est plus question ici de conférer au peuple le don de la théologie. Sa chance, ce sera de trouver sur son chemin des prêtres et des évêques qui lui expliquent l'Évangile comme il faut. La lettre à Paul Volz est donc moins novatrice que l'Exhortation au lecteur pieux. Remarquons également qu'elle ne dit pas un mot de la place et de la fonction des théologiens. Sont-ils assimilés aux prêtres ? Cela est possible, mais, dans ce cas, cela voudrait dire que ce n'est pas le savoir en tant que tel qui donne autorité, mais le ministère, et même le sacrement : celui de l'ordination. Enfin il faut se demander où Érasme se place lui-même dans ce schéma grandiose. Il n'est peut-être pas imprudent de dire qu'il se situe dans le premier cercle, celui des prêtres. Prêtre, il l'est en effet depuis $1492^{18}$. Et il est trop bon théologien (quoi qu'il dise !) pour ignorer qu'on est prêtre pour l'éternité. Son premier testament commence par ces mots : «Moi, Érasme de Rotterdam, docteur en théologie et prêtre $d u$ diocèse d'Utrecht $»^{19}$. Tout le reste n'est qu' affaire de discipline ecclésiastique : délices des biographes qui occultent l'essentiel.

La question que nous nous sommes posée reçoit ici un début de solution. Le discours d'Érasme, plus souvent qu'on ne l'a dit, est un discours sacerdotal. C'est de là qu'il tire la plus grande partie de son autorité, renforcée bien sûr par celle que lui donne son immense culture profane. Il fait partie du premier cercle, et n'a rien à envier aux évêques et aux cardinaux. Mais cette appartenance au premier cercle lui donne plus de devoirs que de privilèges car il doit communiquer à ceux qui sont plus loin du Christ la lumière et le feu provenant de lui.

La Correspondance avec Guillaume Budé, éditée et traduite avec beaucoup de soin par M.-M. de la Garanderie ${ }^{20}$, nous permet d'aller un peu plus loin et de voir comment Érasme assume de facto une partie du rôle de l'intellectuel moderne. L'aspect psychologique d'une relation difficile, où l'amitié, l'admiration et la jalousie dessinent des figures complexes, ne peut faire oublier les questions très importantes que se posent les deux hommes. À plusieurs reprises, Budé range Érasme parmi les théologiens. Hommage ou piège ? Difficile de le savoir. Un théologien, selon Budé, ne doit pas se départir d'une certaine gravité stylistique, d'une certaine hauteur, d'un certain hermétisme. Les mystères de l'Écriture ne doivent être révélés qu'à une minorité. Et de citer les Proverbes de Salomon : "C'est la gloire de Dieu de voiler son langage $»(25,2)^{21}$. Contemporaine de l'Exhortation, la réponse d'Érasme à Budé explique clairement la raison de leur désaccord : « Toi, tu as préféré n'être entendu que des érudits, moi, autant que possible, du plus grand nombre [...]. Toi, tu t'es proposé de convaincre, moi d'ins- 
truire et de persuader $»^{22}$. Tout le reste, y compris les discussions plus nettement stylistiques, vient de là. Scandale : voilà un clerc qui écrit pour être compris et qui, de plus, est persuadé que la grandeur de Dieu n'a rien à y perdre. Sans tomber dans une vision progressiste de l'histoire, dont on connaît les inconvénients, force est de constater qu'Érasme est tourné vers l'avenir, et Budé vers le passé. Le piège est déjoué : Érasme, qui semble vraiment dans cette Correspondance plus fin que Budé, accepte qu'on lui donne le titre de théologien, mais à condition qu'il soit libre de garder le langage qui lui plaît, un langage fait pour toucher le plus grand nombre. Voilà une attitude d'intellectuel, au sens moderne du terme.

D'autant qu'au même moment, Érasme récuse une recommandation de Budé. Désolé de la querelle qui est née entre Lefèvre d'Étaples et Érasme ${ }^{23}$, il exhorte celui-ci à abandonner toute polémique avec lui : " Ainsi l'exige le nom d'Érasme, si réputé et dont résonnent aujourd' hui toutes les provinces d'Occident; ainsi l'exigent et la dignité du prêtre, et l'état de théologien ». ${ }^{24}$ Pour Budé, le débat est indigne du théologien. Érasme est d'un avis différent. Quoi qu'il lui en coûte, mais parfois aussi avec un plaisir évident, il débat et polémique. Car la discussion est inséparable de la persuasion. C'est pourquoi son statut ne peut se réduire ni à celui de théologien tel que l'imagine Budé, ni à celui de philologue où on l'enferme parfois de nos jours. Ici encore, il préfigure l'intellectuel moderne.

Il est bien possible enfin que son refus d'enseigner au Collège des Lecteurs royaux que François Ir est en train de fonder à Paris s'explique aussi par là. Budé lui fait miroiter, de la part du roi, l'image d'une compagnie illustre où se rencontreraient les grands literati, les éminents eruditi de toute l'Europe. La doctrina (la science), mot sacré pour Budé, y régnerait sans entrave, autant du moins que la Sorbonne le tolérerait. On sait qu'Érasme n'a jamais accepté l'offre du roi de France. Pour rester libre ? Sans doute. Mais aussi pour agir plus efficacement. Les grands écrivains de l'époque, il les connaît, il leur écrit souvent. Il leur doit, non au roi de France, une grande part de son prestige. Avec eux, il est un homme illustre. Et c'est en tant qu' « homme illustre » qu'il va intervenir dans l'« affaire Reuchlin ».

\section{III}

On a beaucoup écrit sur celle-ci ${ }^{25}$ et l'on sait qu'Érasme a pris fait et cause pour le grand hébraïsant allemand. Plusieurs spécialistes ont pourtant fait remarquer, avec raison, que le soutien qu'il lui a accordé n'a pas été constant, et que, d'autre part, il a été gêné par l'idée qu'il se faisait du judaïsme ${ }^{26}$. C'est un juif converti, Pfefferkorn, qui fut, à Cologne, l'adversaire le plus coriace de Reuchlin. Aux yeux d'Érasme, son but était tout à fait clair : semer la zizanie chez les chrétiens. Il a donc craint que Reuchlin, dont la foi, à ses 
yeux, était hors de cause, ne soit, à son insu, l'instrument d'un noir complot. Ce qui reste encore à étudier, dans cette affaire, ce sont les raisons qui poussent une grande partie des humanistes de l'époque à défendre leur ami allemand. De quel combat s'agit-il ? Au nom de quoi est-il mené ?

Pour tous, le doute n'est pas permis : Reuchlin est un grand savant. Sa connaissance de l'hébreu, de la Kabbale et du Talmud permet de le considérer comme l'un des représentants les plus éminents de l'humanisme de son temps. C'est en ce sens qu'Érasme écrit au pape Léon X et aux cardinaux Riario et Grimani. Au premier, il explique que Reuchlin «également expert dans les trois langues, grecque, latine, et hébraïque » l'a beaucoup aidé dans son édition du Nouveau Testament». Il ajoute : «Ainsi, c'est à bon droit que toute l'Allemagne tient les yeux levés vers lui, et vénère cet homme comme s'il était un phénix et comme sa seule parure ${ }^{27}$. À cette date, l'affaire est entre les mains du pape ${ }^{28}$. La lettre d'Érasme possède donc la plus grande importance. Alerter Riario et Grimani, c'est aussi de bonne politique. Auprès de l'un comme de l'autre, Érasme plaide la cause de son ami avec éloquence, leur expliquant qu'il a été victime de la jalousie, et que tous les «doctes » allemands se désolent de l'injuste procès qui lui est fait. La bienveillance du pape a l'égard de Reuchlin lui vaudrait la reconnaissance de tous les lettrés : argument auquel le fils de Laurent de Magnifique ne saurait être insensible. Bien d'autres lettres d'Érasme tiennent le même langage Alors même qu'il prend ses distances avec certains défenseurs trop zélés de Reuchlin, Érasme continue d'affirmer qu'il a été injustement attaqué et que tous les hommes savants et honnêtes doivent le soutenir ${ }^{29}$. "Que les doctes (docti) favorisent Reuchlin, c'est un simple acte d'humanité ${ }^{30}$. Érasme souligne aussi le fait que, outre son iniquité, le procès qu'on lui intente trouble le repos de ce grand savant, qui ne peut plus se consacrer en paix aux travaux des Muses ${ }^{31}$.

Jusqu'où va le soutien d'Érasme ? Une première limite se trouve indiquée dans la lettre à Hermann de Neuenahr que nous avons déjà citée. Les hommes savants, explique Érasme, c'est-à-dire les intellectuels, ne doivent pas se commettre avec des fantoches ou des monstres comme Pfefferkorn ${ }^{32}$, le dominicain exécré par Érasme. Sans le savoir, ils font de la publicité pour un individu sinistre, trop content d'être placé sous les feux de la rampe par des humanistes un peu naïfs. Les eruditi devraient avoir une idée d'eux-mêmes qui les empêchât de se commettre avec des gens ignobles. Pirckheimer est du même avis. Il rappelle à Reuchlin, dans une lettre de décembre 1512, que dans l'antiquité, les Éphésiens avaient interdit de prononcer le nom de l'incendiaire du temple de Diane afin que la gloire ne soit pas la récompense de son crime. Reuchlin devrait s'inspirer de son exemple, lui qui par ses livres fait connaître un homme obscur ${ }^{33}$. Ce discours 
explique les réserves de certains humanistes qui ne sont pas tous sur la brèche pour défendre Reuchlin.

Celui-ci, au reste, hésite quelque peu sur l'attitude qu'il doit adopter devant ses adversaires. Redoutable dans la satire, comme on peut le voir avec l'Augenspiegel, il semble parfois, dans sa Correspondance, opter pour la non-résistance. Pirckheimer la lui conseille ; un homme comme lui ne doit pas craindre les attaques des Thersite et des Zoïle ; il se souvient que le sage est toujours en butte à l'envie ${ }^{34}$. Plus intéressant encore est un échange de lettres entre Cuspinien et Reuchlin, qui date du printemps 1512. Le premier exhorte son ami à regarder de haut la folie de ses adversaires ${ }^{35}$. Le second abonde dans son sens et répond que, de fait, il ne peut rien arriver à un homme de bien, de son vivant ou après sa mort : « Socrate est vivant pour l'éternité, il triomphe dans la gloire, alors que la mémoire de ses adversaires est totalement éteinte ${ }^{36}$. L'image de Socrate tend à faire de Reuchlin un martyr de la vérité, l'un de ces justes qui paient de leur mort leur zèle pour celle-ci. Heureux ceux qui souffrent persécution pour la justice! Dans la lettre que nous commentons, la citation des Béatitudes suit très logiquement l'évocation de Socrate : «Ego vero beatus futurus sum quem persequuti sunt propter justitiam ». À quoi bon se tourmenter, expliquent certains correspondants de Reuchlin : la vérité triomphera d'elle-même.

Elle ne dédaigne pas, cependant, l'aide que l'on peut lui apporter. Il y a eu un véritable "parti Reuchliniste », où l'on retrouve le gotha des intellectuels de l'époque. On le sait d'abord par Érasme qui ne veut pas en être : «Je ne suis pas Reuchliniste et n'appartiens à aucune faction ${ }^{37}$. À cette date (1519), les « Reuchlinistes », s'étaient fait connaître de bruyante façon. Ils avaient célébré le triomphe de leur héros, quand celui-ci avait été acquitté par le tribunal ecclésiastique de Spire ${ }^{38}$, et une gravure contemporaine le représentait entrant dans sa bonne ville de Pforzheim « sur un char de triomphe à la romaine, couronné de laurier et de lierre, entouré d'amis, de musiciens et de choristes : dans sa main droite, il tient l'Augenspiegel et dans la gauche une branche de laurier. Des allégories de la Superstition, de l'Envie, de la Barbarie et de l'Ignorance, ainsi que les figures d'Hochstraten [l'inquisiteur], de Pfefferkorn et Ortwin Gratius, suivent le char à pied et enchaïnées ${ }^{39}$.

Plus significatif encore pour notre sujet est le premier recueil des Clarorum virorum Epistolae, publié à Tubingen en $1514^{40}$. Il s' agissait d'un choix de lettres adressées à Reuchlin par des hommes célèbres de la République des Lettres. Comme le souligne Geiger ${ }^{41}$, ce n'était pas une initiative des amis de Reuchlin, mais une idée de celui-ci, ce qui appert de ces mots d'Hiltebrant : "Jean Capnion nous a apporté cet élégant volume où se trouvent rassemblées un certain nombre de lettres d'hommes très célèbres », 
qui lui étaient favorables ${ }^{42}$. Cela risque fort d'être une première dans l'histoire des idées en Occident. Un écrivain, inquiété par les tribunaux et attaqué par de redoutables adversaires, fait intervenir l'opinion publique et manifeste l'estime dans laquelle le tiennent les «intellectuels » en publiant les lettres qu'ils lui ont adressées. Le volume, préfacé par Melanchthon, est prestigieux. Il l'aurait été encore davantage si on avait pu y trouver une ou plusieurs lettres d'Érasme. Mais les relations épistolaires des deux hommes ne commencent qu'après cette publication, et à l'initiative de l'Allemand qui s'adresse à Érasme pour la première fois en avril $1514^{43}$. Ce n'est que partie remise. Un second volume de Lettres d'hommes célèbres (Illustrium virorum Epistolae) est publié à Haguenau en mai 1519, chez l'éditeur Anshelm. Y figurent quatre lettres d'Érasme : $n^{\text {os }} 300,324,457$ et 471 . Ce volume est encore plus intéressant que le précédent, car on voit y apparaître ce que l'on peut vraiment appeler un «parti du savoir». Une liste des défenseurs de «Capnion » (c'est-à-dire Reuchlin) regroupe plus d'une vingtaine de noms, «viri Germaniae totius literatissimi splendidissimique ${ }^{44}$. À tout seigneur tout honneur : le premier nommé est Érasme «vir seculi nostri doctissimus ». On trouve ensuite les noms de Hutten, Capiton, Oecolampade, Peutinger, Mutianus Rufus, Pirckheimer, Vadianus, Lazius, Cuspinien, Melanchthon et bien d'autres encore ${ }^{45}$. D'autres listes circulent. Dans une édition de Lucien, Pirckheimer avait constitué la sienne en incluant le nom d'Érasme, ce qui n'avait pas eu l'heur de plaire au Rotterdamois qui le lui fit savoir ${ }^{46}$. Les Allemands ne sont pas les seuls à soutenir Reuchlin. Dans l'une des éditions partielles de la Correspondance d'Érasme (Farrago nova epistolarum, 1519), la lettre 471 d'Érasme à Reuchlin est suivi d'un post scriptum anonyme commençant par les mots suivants : "voici quels sont en Angleterre les très savants amis de Reuchlin ». Dans la liste qui suit, on trouve notamment les noms de J. Colet, et Thomas More. Heureux Reuchlin, qui a de tels amis ! Michelet avait donc raison : le $\mathrm{XVI}^{\mathrm{e}}$ siècle, c'est la lutte de la science contre l'ignorance. Figure emblématique de celle-ci : Reuchlin, entouré de ses amis.

\section{IV}

Il faut pourtant regarder les choses de plus près. Quand Érasme, de plus ou moins bonne grâce, intervient en faveur de Reuchlin, il a soin de souligner que celui-ci n'est pas un homme seul et qu'il peut compter sur d'autres soutiens que ceux des intellectuels. "Il est bien vu, écrit-il à Riario, de l'empereur Maximilien, au point qu'il fait partie de ses conseils ${ }^{47}$. L'exactitude de l'information ne l'empêche pas d'être signifiante. Érasme veut faire comprendre au cardinal que les Dominicains se sont attaqués peut-être à forte partie. Les listes de partisans de Reuchlin ne disaient pas autre chose. 
Mêlés aux noms des écrivains célèbres figuraient en effet ceux de personnages puissants et respectés. Juste après Érasme, on trouvait celui de «Hermannus comes de Neunar, nobilium omnium specimen ». Les conseillers de Maximilien ne manquaient pas non plus. Même si l'imagerie romantique doit en souffrir, il faut dire nettement que Reuchlin n' est pas seul contre tous. Il a des alliés haut placés dans la lutte contre le monstre Ignorance. D'ailleurs les écrivains pressent ceux-ci d'intervenir pour soutenir l'une des gloires de l'Allemagne et faire cesser les aboiements des Dominicains. À plusieurs reprises, Érasme s'étonne de la passivité des autorités civiles. Pourquoi les magistrats somnolent-ils ? "Ils laissent cette peste délirer impunément contre des hommes érudits et célèbres (in eruditos et claros homines) ». Il faudrait un Hercule pour précipiter de tels monstres dans les ténèbres infernales. "C'est ainsi, et non par des livres, qu' on peut les vaincre » ${ }^{48}$. On ne saurait faire plus clairement appel au bras séculier.

L'attitude de Reuchlin justifie le discours de ses partisans. Si, d'un côté, il lui arrive de poser en martyr de la vérité, de l'autre, il recherche l'alliance du pouvoir et du savoir. La lettre qu'il adresse à Léon X en mars 1517 est tout à fait significative. Elle commence par un grand éloge des Médicis et en particulier de Côme l'Ancien, sous qui Florence brilla de mille feux. Reuchlin énumère les gloires de son règne : Pic de la Mirandole, Ficin, Politien et bien d'autres. Il résume ensuite le conflit qui l'oppose aux Dominicains de Cologne. Tout cela est de bonne politique. Ce qui est plus étonnant est la manière dont il essaie de faire pression sur le pape : il a pour lui, explique-t-il, beaucoup d'Allemands très respectables ; son innocence, sa bonne foi, sa piété sont garanties par des princes, des magistrats et des évêques. Les meilleurs témoins de l'humaniste ne sont donc pas, dans cette lettre, des savants, mais ceux qui détiennent une part de l'autorité temporelle $^{49}$. On voit où tend cette argumentation : le pape, confiant dans ces témoignages, doit protéger Reuchlin comme ses ancêtres ont protégé les écrivains florentins de leur temps. Ce langage, on le trouvait déjà, quelque temps auparavant, dans une lettre à Mutianus Rufus. L'humaniste allemand y expliquait qu'il n'était pas défendu seulement par la vérité, mais par des personnages puissants qui ont horreur de la calomnie ${ }^{50}$.

Pour être encore mieux déniaisés, il nous faut enfin relever un passage très étonnant d'une lettre de Pirckheimer adressée à Neuenahr ${ }^{51}$. Elle fait intervenir un épisode dramatique de l'histoire florentine : celui de Savonarole $^{52}$, auquel Érasme fait lui aussi allusion ${ }^{53}$. Une lecture à la fois romantique et pressée identifierait vite les deux figures de persécutés. Elle se tromperait lourdement. Savonarole n'est pas le prototype de Reuchlin, mais celui de l'inquisiteur Hochstraten. L'un comme l'autre sont des dominicains. L'un comme l'autre croient de leur devoir de défendre la cause de la vérité 
à temps et à contre-temps. L'Inquisiteur avait souvent, semble-t-il, un verset d'Isaïe $(62,1)$ à la bouche : pour la cause de Sion, il ne se tiendrait pas tranquille, quitte à désobéir à l'empereur qui avait ordonné aux deux parties de se taire en attendant la décision du pape. D'un côté comme de l'autre, désobéissance : l'Inquisiteur désobéit à l'empereur comme Savonarole avait désobéi au pape. On réclame finalement contre le premier l'action du bras séculier.

Voilà où en sont nos humanistes. Ils ne croient pas tellement aux droits de la vérité toute seule. Ils l'applaudissent quand elle est appuyée par des hommes considérables et quand ses protagonistes sont les familiers des puissants de ce monde. Nos intellectuels hésitent singulièrement à s'engager dans les combats douteux de l'histoire. Les docti de l'époque imaginent peut-être la première pétition de l'histoire de l'Occident. Mais, pour Érasme comme pour les autres, le savoir sans le pouvoir est finalement peu de chose.

\section{V}

Passablement irrité par le tour que prend l'affaire Reuchlin, Érasme le devient encore plus quand sont publiées les Epistolae obscurorum virorum (Épitres des hommes obscurs). Une première partie apparaît en octobre 1515. Une seconde voit le jour en 1517 , tandis que la première connaît d'autres éditions augmentées ${ }^{54}$. Le mécontentement de l'humaniste est exprimé dans plusieurs lettres, mais les raisons invoquées, comme toujours, ne sont pas les seules, ni même les plus importantes. À plusieurs reprises (lettres 622 et 626), il regrette les attaques personnelles lancées par Hutten et ses complices. Cela, on ne peut le contester. Les auteurs s'en prennent à Hochstraten, Pfefferkorn, et à tout un peuple d' " hommes obscurs », qui est bien réel ${ }^{55}$. Toutes ces lettres fictives sont adressées à l'un des principaux représentants du parti anti-reuchliniste : «Notre maître » Ortvinus Gratius. Le moins que l'on puisse dire, est que le portrait de ces « hommes obscurs » n'est pas flatté. Plus ignorants les uns que les autres, buvant sans vergogne et forniquant de la même manière, ils annoncent, en plus noir, le personnage de Janotus de Bragmardo. Rabelaisien avant la lettre, aussi, le procédé satirique choisi par les auteurs anonymes et qui consiste à laisser les personnages se ridiculiser eux-mêmes. Tout cela dans un joyeux latin macaronique, où l'on retrouve l'esprit du carnaval et celui des farces étudiantes ${ }^{56}$. Même s'il ne le dit pas ouvertement, il est clair que ni le ton ni le style ne plaisent à Érasme. L'Éloge de la Folie, c'était autre chose ${ }^{57}$. Le mécontentement d'Érasme est d'autant plus vif, qu'on lui attribue la paternité de l'ouvrage (ép. 808), et que, dans la seconde partie, publiée en 1517, il est très souvent cité. Or, rien ne déplaît autant à l'humaniste que de voir son image lui échapper. 
Un auteur moins fin que lui se serait plu aux éloges qui lui sont adressés. Il apparaît comme un nouveau Pic de la Mirandole : " homo valde doctus in omni scibili omnique doctrinarum genere $»^{58}$. «A multis honoratur ${ }^{59}$. Il a écrit au pape pour soutenir Reuchlin, et les « hommes obscurs » redoutent son savoir et son influence. Cependant, et au moins à deux reprises, Érasme est présenté comme un poète plus que comme un théologien (II, 38 et II, 49). Plus grave encore : l'auteur de la lettre II, 49 suggère que son titre de théologien est usurpé : "Super hoc est hic unus theologus, ut ipse se nominat, sed mihi videtur, quod est magis poeta $»^{60}$. Bien entendu, c'est un « homme obscur» qui est censé parler. Mais de la part de Hutten et de ses amis, il s'agit d'une double "gaffe». Ils ont le tort de semer le doute sur la réalité du titre de théologien acquis, comme on l'a vu, par Érasme à Turin. En outre, ils ont l'air d'ignorer que l'illustre humaniste l'exhibe rarement. On comprend l'intention des auteurs : discréditer la théologie scolastique qui ne jure que par Aristote et ses commentateurs et par la disputatio en bonne et due forme. Ni Érasme ni Reuchlin ne se sont livrés à ce genre d'exercice « sicut est mos ${ }^{61}$. Un personnage rencontré par l'auteur de la lettre II, 50 défend leur conception de la théologie et qualifie de «sophistique » la théologie des gens de Cologne ${ }^{62}$. Il n'empêche : les auteurs des Epistolae obscurorum virorum ont été imprudents.

À leur insu, peut-être, ils accréditent aussi l'idée que tout le monde peut faire de la théologie. Tout le monde écrit des livres qui relèvent de celle-ci : « omnes jam scribunt libros in theologia secundum suum libitum »63. Fallait-il donner l'impression que les " hommes obscurs », pour une fois, ne sont pas ridicules? Quand l'un d'entre eux défend l'idée que la théologie réclame des compétences et que l'on doit rester dans l'état qui est le sien, il est loin d'avoir tort, même si son style est quelque peu risible : «Unusquisque debet manere in facultate sua, et non debet mittere falcem in messem alterius; quia sutor est sutor, et sartor est sartor, et faber est faber. Et non staret bene, si unus sartor vellet facere calceos ${ }^{64}$. En s'appuyant sur la vieille sagesse des nations, les « hommes obscurs » imaginés par Hutten et ses amis risquent d'avoir raison plus qu'il n'est souhaitable. Le mécontentement d'Érasme dut être d'autant plus grand qu'il avait soutenu l'idée que tout le monde, en somme, pouvait être théologien, s'il lisait l'Écriture Sainte avec piété. Le piège se refermait sur lui, et par la faute d'amis maladroits. Ce qu'ils souhaitaient, plus ou moins ouvertement, devait se réaliser quelques années plus tard lorsque, en Allemagne, tout le monde écrivit pour ou contre Luther. D'une certaine façon, l' « affaire Reuchlin » ne fait que préfigurer la Réforme luthérienne. Érasme déplora alors ce qu'il avait donné l'impression d'encourager : tout le monde ne pouvait être théologien ${ }^{65}$. Si 
cette discipline devait se réformer, ce devait être de l'intérieur, non grâce à l'apport des barbiers et des tisserands.

\section{VI}

À bien des égards, l'affaire Reuchlin est fort instructive. Elle nous apprend d'abord qu'il ne faut pas croire les humanistes sur parole. Certes, ils ont défendu leur collègue allemand, mais sans trop se soucier de la question qui était au cœur de l'affaire : celle de la culture hébraïque et des livres qui la perpétuaient. Pour Érasme et pour beaucoup, le nom de Reuchlin est devenu un étendard qu'ils brandissaient dans leur lutte contre les vieilleries médiévales. Il fallait à tout prix «écraser l'infâme ». Le reste, pour beaucoup d'entre eux, était finalement secondaire. En la personne de Reuchlin, les dominicains de Cologne emmenés per Pfefferkorn, leur âme damnée, avaient eu le tort d'attaquer le savoir : crime de lèse-majesté que les docti ne pouvaient pas tolérer. Ils se rebiffent, et rédigent des sortes de pétitions où apparaissent les plus grands noms de l'Europe savante, mêlés à ceux des princes et des puissants de ce monde. Pour eux, le savoir a besoin du pouvoir s'il veut se faire entendre. Le temps où l'Esprit pourrait parler tout seul n'est pas encore venu.

D'une certaine manière, pourtant, il n'est pas loin. On sent déjà chez Érasme un avant-goût de la Réforme et de son prophétisme. Les prophètes, en effet, se moquent bien du savoir. Ils parlent au nom de la vérité dont ils essaient de se tenir proches. La lettre d'Érasme à Paul Volz adopte cette posture. Mais elle n'a pas d'effets durables sur ses engagements. Ce n'est pas en prophète ou en prêtre qu'il prend position dans l'affaire Reuchlin, mais en tant que représentant éminent du nouveau savoir. Comme tel, il ne tient pas en grande estime (c'est le moins qu' on puisse dire) les prophètes de son temps, Savonarole par exemple. Le seul humaniste de son temps qui ait tenu un rôle prophétique, c'est Thomas More. On sait ce qui lui advint. Son destin tragique n'empêcha pas les intellectuels de croire encore longtemps à l'alliance heureuse du savoir et du pouvoir. Il faudra plus de deux siècles pour que cette illusion s'évanouisse.

Université Paris $X$ - Nanterre

\section{Notes}

1. Jean-Paul Sartre, Plaidoyer pour les Intellectuels, Paris, Gallimard, 1972.

2. Ibid., p. 12-13.

3. L. Febvre, Le Problème de l'incroyance au XVIe siècle. La religion de Rabelais, édition revue, coll. «L'Evolution de l'humanité », Paris, Albin Michel, 1947. 
Daniel Ménager / Érasme, les intellectuels et l'affaire Reuchlin / 61

4. Voir Jacques Le Goff, Les Intellectuels au Moyen Âge, Paris, Seuil, 1960.

5. Ibid., p. 4.

6. Voir la conclusion, aussi belle que contestable, de ce livre (Le Goff, p. 187-88).

7. A. Chastel, Le Mythe de la Renaissance, Genève, Skira, 1969, p. 167-75.

8. L.-E. Halkin, Érasme parmi nous, Paris, Fayard, 1987, p. 103.

9. En l'occurrence, Turin. Voir J. Chomarat, Grammaire et rhétorique chez Erasme, Paris, Les Belles Lettres, 1981, p. 17.

10. Érasme, Apologie à J. Lefèvre d'Étaples, ASD IX-3, cité et traduit par Chomarat, op. cit., p. 18.

11. Chomarat, p. 20.

12. Érasme, Épître 393, « À Charles de Brabant » [futur Charles Quint], Correspondance, éd. Gerlo et alii, Bruxelles, De Graaf, 1967-84, t. II, p. 301. Les lettres d'Érasme seront citées dans cette traduction. Quand le texte latin est important, nous le donnons d'après l'édition Allen, Oxford, Clarendon Press, 11 volumes, 1906-58.

13. Au plus fort de la polémique avec Luther, Érasme ne cessera de répéter qu'il n'appartient à aucun parti.

14. Érasme, Exhortation au lecteur pieux (Paraclesis), Les Préfaces au Nouveau Testament, éd. Delègue-Gillet, Genève, Labor et Fides, 1990. La traduction est celle de Chomarat, op. cit., p. 17.

15. C'est la préface de la seconde édition du Manuel du soldat chrétien (Enchiridion militis christiani), publiée en 1518. Voir l'édition bilingue du Père Festugière (Paris, Vrin, 1971), reprise dans Érasme, Paris, R. Laffont, « Bouquins », p. 620-41.

16. Ibid., p. 629-31.

17. Voir l'édition de la Hiérarchie céleste par G. Heill, introduction de R. Roques, étude et texte critique de G. Heil, traduction et notes par M. de Gandillac, Paris, Cerf, 1970. À l'époque d'Érasme, ce texte du $\mathrm{IV}^{\mathrm{e}}$ ou du VI ${ }^{\mathrm{e}}$ siècle après J.-C. exerçait encore une grande influence. Mais le Rotterdamois ne pensait pas, à la différence de ses contemporains, qu'il était l'œuvre du disciple de saint Paul.

18. Voir Halkin, p. 27.

19. Épître 1527, édition «Bouquins », p. 1129.

20. Paris, Vrin, 1967.

21. Épître 493, éd. citée, p. 85. Les traductions modernes, plus fidèles à l'hébreu, traduisent ce verset d'une tout autre façon : «La gloire de Dieu, c'est d'agir dans le mystère » (TOB).

22. Épître 531, 15 février 1517, éd. citée, p. 116.

23. Sur cette querelle, dont l'origine est un point d'exégèse (comment comprendre Heb. 2, 7) et qui commence en 1517, voir G. Bédouelle, Lefèvre d'Étaples et l'intelligence des Écritures, Genève, Droz, 1976.

24. Épître 610, 12 avril 1518, éd. citée, p. 154.

25. Une bonne bibliographie, due à A. Cisek, se trouve dans les Centuriae latinae (Mélanges J. Chomarat), article "Reuchlin », Genève, Droz, 1997, p. 677-78. Voir L. Geiger, Johannes Reuchlin, sein Leben und sein Werk, Leipzig, 1871 ; M. Brod, Johannes Reuchlin und sein Kampf, Kohlhammer Verlag, 1965 ; G. Vallese, Erasmo e Reuchlin, Naples, 1964 ; 
62 / Renaissance and Reformation / Renaissance et Réforme

et M. Krebs, « Reuchlin Beziehungen zu Erasmus von Rotterdam », Johannes Reuchlin (1455-1522), Sigmaringen, Jan Thorbecke Verlag, 1994 (reprint de l'édition de 1955).

26. Voir A. Godin, «L'antijudaïsme d'Erasme », Bibliothèque d'Humanisme et Renaissance, 1984, p. 537-53.

27. Épître 335, Correspondance, éd. Gerlo, t. II, p. 10.

28. Résumons l'essentiel d'une histoire et d'une procédure compliquées. Dans la première décennie du XVI ${ }^{\mathrm{e}}$ siècle, le dominicain de Cologne, J. Pfefferkorn, juif converti, entame une violente campagne contre le judaïsme et demande que l'on brûle tous les livres écrits en hébreu. L'empereur Maximilien consulte quelques experts, dont J. Reuchlin, connu pour ses travaux sur le Talmud et la Kabbale. Le 6 octobre 1510, il formule un avis hostile à Pfefferkorn. Commence alors une longue polémique avec le dominicain et des membres de son ordre. Pfefferkorn publie le Handsspiegel. Reuchlin réplique avec l'Augenspiegel (1511), qui sera suivi de la Defensio (1512). Une procédure inquisitoriale, menée par Hochstraten, est mise en place. En 1513, Reuchlin fait appel au pape. L'année suivante, il est acquitté par le tribunal ecclésiastique de l'évêque de Spire. Hochstraten fait appel de ce jugement à Rome, tandis que Reuchlin rassemble ses défenseurs dans le premier volume des Clarorum virorum Epistolae (mars 1514). Crotus Rubeanus et Ulrich von Hutten, l'un et l'autre partisans de Reuchlin, font paraître en octobre 1515 le premier volume des Epistolae obscurorum virorum, recueil de lettres fictives qui ridiculisent les adversaires de l'humaniste. Un deuxième suivra en 1517. Finalement, Reuchlin est condamné à Rome en 1520, mais cette condamnation n'eut pas beaucoup d'effet.

29. Épître 703, adressée à Hermann de Neuenahr, l'un des plus fermes soutiens de Reuchlin, Correspondance, éd. Gerlo, t. III, p. 152-53.

30. Épître 701, Correspondance, éd. Gerlo, t. III, p. 151.

31. Voir, par exemple, l'épître 333.

32. Épître 703, Correspondance, éd. Gerlo, t. III, p. 153.

33. Pirckheimer, Briefwechsel, Munich, C.H. Beck, 1940-1997, épître 234, t. II, p. 211.

34. Ibid., épître 197, p. 112.

35. Reuchlin, Briefwechsel, Stuttgart, 1875, reprint G. Olms, 1962, épître CXLVII, p. 169.

36. Ibid., épître CXLVIII, p. 172.

37. Épître 1041, Correspondance, éd. Gerlo, t. IV, p. 133.

38. Voir Geiger, p. 290 et suiv., et Krebs, p. 143.

39. Notice « Reuchlin », Centuriae latinae (Mélanges J. Chomarat), Genève, Droz, 1997, p. 669.

40. Geiger, p. 322-23.

41. Ibid., p. 323.

42. Ibid., note 5, p. 322-23. Nous avons traduit du latin la phrase que nous citons.

43. Allen et Gerlo, épître 290 ; voir Krebs, p. 142 et notes 12 et 13.

44. Un fac-simile de cette liste se trouve dans l'article de Krebs, p. 148.

45. Sur les personnalités moins connues de cette liste, voir Peter G. Bietenholz, éd., Contemporaries of Erasmus: A Biographical Register of the Renaissance and Reformation, 3 vol., Toronto, University of Toronto Press, 1985-87. 
46. Voir l'épître 694 d'Erasme. La liste de Pirckheimer figure dans une longue lettre du 30 août 1517, Briefwechsel, ép. 464, t. III, p. 162.

47. Épître 333, Correspondance, éd. Gerlo, t. II, p. 88.

48. Épître 703, Correspondance, éd. Gerlo, t. III, p. 153.

49. Briefwechsel, épître CCXXXVIII, mars 1517, éd. citée, p. 273-74.

50. « Non sola veritate [. . .], verum etiam a potentissimis hominibus, qui et veritatem amant, et oderunt calumnias » (épître CLXVIII, ibid., p. 192).

51. Briefwechsel, épître 540, t. III, p. 332.

52. Il serait très intéressant d'étudier l'image de Savonarole dans le premier humanisme. A notre connaissance, cette étude n'a pas encore été faite. Elle serait sans doute riche de surprises.

53. Épître 694, Correspondance, éd. Gerlo, t. III, p. 140.

54. Voir l'introduction d'Aloys Bömer, dans son édition des Epistolae obscurorum virorum, 2 vol., Heidelberg, R. Weissbach, 1924, t. I.

55. Ibid., p. 6-48.

56. Ibid., p. 65-74.

57. Érasme, épître 636.

58. Epistolae obscurorum virorum (nous abrégerons ce titre en EOV), édition Bömer, I, 72. L'auteur de cette lettre fictive situe sa rencontre avec Érasme lors du voyage triomphal que fit celui-ci à Strasbourg, en 1514 : voir Halkin, p. 142.

59. EOV, II, 38, p. 155.

60. Ibid.

61. Ibid., II, 33, p. 148.

62. Ibid., II, 50, p. 174.

63. Ibid., I, 48, p. 85.

64. Ibid., I, 15, p. 30.

65. Cette polémique se développa après l'affrontement direct avec Luther, dans les années 1525-26. L'idée eut un bel avenir et elle servit de nouveau au début des guerres civiles en France, quand les poètes catholiques croisèrent le fer avec les protestants. Ronsard l'orchestra avec brio : voir la «Remonstrance au peuple de France », v. 187-90, Pierre de Ronsard, Eeuvres complètes, éd. Jean Céard, Daniel Ménager, et Michel Simonin, 2 vol., «Bibliothèque de la Pléiade », Paris, Gallimard, 1993-94, t. II, p. 1024. 